\title{
Soft-Information Assisted Near-Optimum Nonlinear Detection for BLAST-type Space Division Multiplexing OFDM Systems
}

\author{
M. Jiang, Student Member, IEEE, J. Akhtman, Student Member, IEEE, and Lajos Hanzo, Fellow, IEEE
}

\begin{abstract}
In this contribution, a nonlinear hybrid detection scheme based on a novel soft-information assisted Genetic Algorithm (GA) is proposed for a Turbo Convolutional (TC) coded Space Division Multiplexing (SDM) aided Orthogonal Frequency Division Multiplexing (OFDM) system. Our numerical results show that the performance of the currently known GA-assisted system can be improved by about $2 \mathrm{~dB}$ with the aid of the GA's population-based soft solution, approaching the optimum performance of the soft-information assisted Maximum Likelihood (ML) detection, while exhibiting a lower complexity, especially in high-throughput scenarios. Furthermore, the proposed scheme is capable of achieving a good performance even in the so-called overloaded systems, where the number of transmit antennas is higher than the number of receiver antennas.
\end{abstract}

Index Terms-Genetic algorithm, orthogonal frequency division multiplexing, soft information, space division multiplexing,

\section{INTRODUCTION}

$\mathbf{R}$ ECENTLY, Genetic Algorithm (GA) [1] assisted MultiUser Detection (MUD) techniques have been proposed for both multi-user Code Division Multiple Access (CDMA) [2]-[5] as well as Space Division Multiple Access (SDMA) type uplink Orthogonal Frequency Division Multiplexing (OFDM) [6], [7] systems. However, to the best of our knowledge all GA-based detection schemes found in the open literature at the time of writing are only capable of providing a hard-decision output for the channel decoder, which inevitably limits the system's achievable performance. Against this background, we propose the novel concept of a GA-aided detection scheme benefitting from populationbased soft solutions and quantify its performance gain in a Turbo Convolutional (TC) coded Multiple-Input-MultipleOutput (MIMO) OFDM architecture, which is referred to here as Space Division Multiplexing (SDM) assisted OFDM. We will demonstrate that the proposed Bell Labs Layered SpaceTime (BLAST) [8] type SDM-OFDM system exploiting the GA's soft outputs is capable of outperforming its counterpart based on hard-decision outputs, while achieving a similar

Manuscript received July 27, 2005; revised December 20, 2005; accepted January 3, 2006. The associate editor coordinating the review of this letter and approving it for publication was E. Serpedin. The work reported in this paper has formed part of the Wireless Enablers 2.2 of the Core 3 Research Programme of the Virtual Centre of Excellence in Mobile and Personal Communications, Mobile VCE, www.mobilevce.com, whose funding support, including that of EPSRC, is gratefully acknowledged. Fully detailed technical reports on this research are available to Industrial Members of Mobile VCE.

M. Jiang was with the School of Electronics and Computer Science, University of Southampton, SO17 1BJ, UK. He is now with Samsung (email: ming.jiang@ samsung.com).

J. Akhtman and L. Hanzo are with the School of Electronics and Computer Science, University of Southampton, SO17 1BJ, UK (email: yja02r@ecs.soton.ac.uk; lh@ecs.soton.ac.uk).

Digital Object Identifier 10.1109/TWC.2007.05578

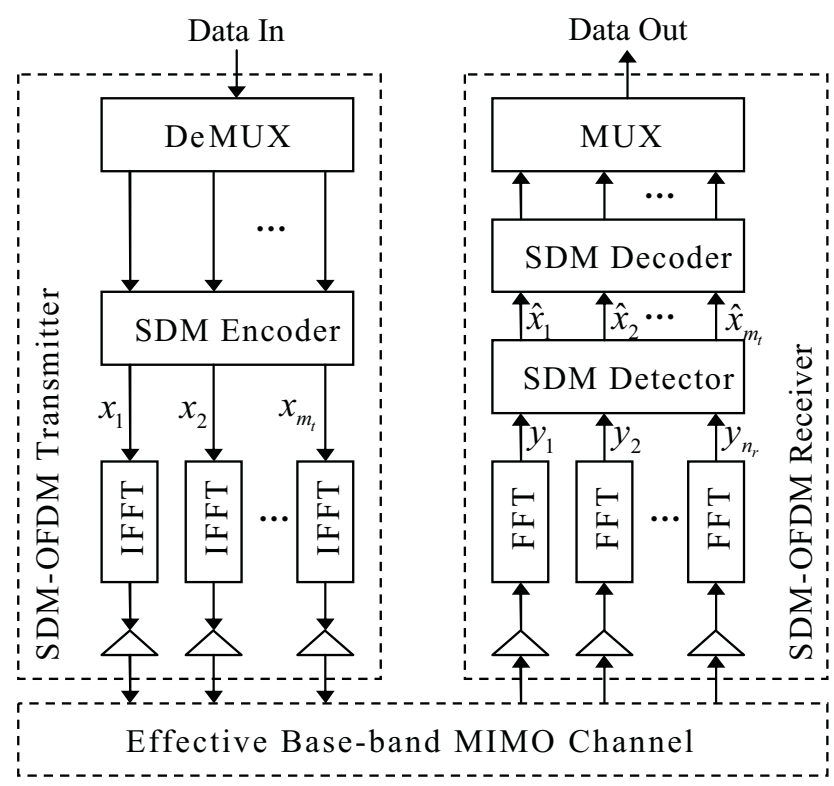

Fig. 1. Schematic of a SDM-OFDM BLAST-type transceiver.

performance to that attained by soft-decision assisted optimum Maximum Likelihood (ML) detection. Furthermore, the proposed scheme is capable of achieving a good performance even in the so-called overloaded scenarios, where the number of transmit antennas is higher than the number of receiver antennas. Finally, the computational complexity of the proposed scheme is significantly lower than that of the optimum ML system, especially in high-throughput scenarios.

The structure of this paper is as follows. The SDM-OFDM system model is introduced in Section II, followed by a brief review of the conventional-GA assisted detection technique in Section III. The proposed GA detection with population-based soft outputs is detailed in Section IV. Our numerical results are presented in Section V, while Section VI concludes our findings.

\section{SDM-OFDM ARCHITECTURE}

Here the terminology of SDM is used for the sake of differentiating it from the conventional BLAST scheme [8], which usually refers to the point-to-point single-carrier MIMO architecture. The discrete-time model of the SDM-OFDM MIMO system is illustrated in Fig. 1, which can be characterized by:

$$
y_{i}[n, k]=\sum_{j=1}^{m_{t}} H_{i j}[n, k] x_{j}[n, k]+w_{i}[n, k],
$$


where $n=0,1, \ldots$, and $k=0, \ldots, K-1$ are the OFDM symbol and subcarrier indices, respectively, while $y_{i}[n, k], x_{j}[n, k]$ and $w_{i}[n, k]$ denote the symbol received at the $i^{t h}(i=$ $\left.1, \ldots, n_{r}\right)$ receive antenna, the symbol transmitted from the $j^{\text {th }}\left(j=1, \ldots, m_{t}\right)$ transmit antenna and the Gaussian noise sample encountered at the $i^{\text {th }}$ receive antenna, respectively. Furthermore, $H_{i j}[n, k]$ represents the complex-valued Channel Transfer Function (CHTF) associated with the propagation link connecting the $j^{t h}$ transmit and the $i^{t h}$ receive antennas at the $k^{\text {th }}$ OFDM subcarrier and time instance $n$. For the sake of mathematical convenience, Equation (1) can be expressed in the form of vectors and matrices, as:

$$
\mathbf{y}[n, k]=\mathbf{H}[n, k] \mathbf{x}[n, k]+\mathbf{w}[n, k],
$$

where we introduce the space-division related vectors $\mathbf{y}, \mathbf{x}$ and $\mathbf{w}$, as well as the $\left(n_{r} \times m_{t}\right)$-dimensional CHTF matrix $\mathbf{H}$, as follows:

$$
\begin{aligned}
\mathbf{y} & =\left(y_{1}, y_{2}, \ldots, y_{n_{r}}\right)^{T} \\
\mathbf{x} & =\left(x_{1}, x_{2}, \ldots, x_{m_{t}}\right)^{T}, \\
\mathbf{w} & =\left(w_{1}, w_{2}, \ldots, w_{n_{r}}\right)^{T}, \\
\mathbf{H}= & \left(\begin{array}{cccc}
H_{11} & H_{12} & \cdots & H_{1 m_{t}} \\
H_{21} & H_{22} & \cdots & H_{2 m_{t}} \\
\vdots & \vdots & \ddots & \vdots \\
H_{n_{r} 1} & H_{n_{r} 2} & \cdots & H_{n_{r} m_{t}}
\end{array}\right) .
\end{aligned}
$$

Note that in Equations (3) to (6) the notation of $[n, k]$ has been omitted for brevity, but the above equations refer to any of the $K$ subcarriers in the $n^{t h}$ OFDM symbol.

\section{GA-BASED SDM DETECTION}

It is well known that the optimum ML detection [9] uses an exhaustive search for finding the most likely transmitted signals. For the SDM-OFDM system employing $m_{t}$ transmit antennas, the ML detection requires a total of $M=\left(2^{m}\right)^{m_{t}}$ metric evaluations, where $m$ denotes the number of bits per symbol (BPS), in order to detect the symbol vector $\hat{\mathbf{x}}_{\mathrm{ML}}$ that consists of the most likely transmitted $\left(2^{m}\right)$-ary symbols of the $m_{t}$ transmit antennas at a specific subcarrier. Explicitly, the ML complexity increases exponentially with both the number of BPS and the number of transmit antennas $m_{t}$. By contrast, the GA-based detection schemes [2]-[7] confine their search to a small fraction of the ML detector's search space by evaluating a system-dependent Objective Function $(O F)$ at a substantially reduced number of up to $X \cdot Y \ll M$ times, where $X$ is the GA's population size and $Y$ is the number of GA generations. In the context of the SDM-OFDM system, the OF can be formulated according to the ML decision metric as:

$$
\Omega(\check{\mathbf{x}})=\|\mathbf{y}-\mathbf{H} \check{\mathbf{x}}\|^{2},
$$

where $\check{\mathbf{x}}$ is a trial-vector in the set $\mathcal{M}^{m_{t}}$, which has a size of $M$ and is defined by:

$\mathcal{M}^{m_{t}}=\left\{\check{\mathbf{x}}=\left(\check{x}_{1}, \check{x}_{2}, \ldots, \check{x}_{m_{t}}\right)^{T} \mid \check{x}_{1}, \check{x}_{2}, \ldots, \check{x}_{m_{t}} \in \mathcal{M}_{c}\right\}$,

and $\mathcal{M}_{c}$ denotes the set containing the $2^{m}$ number of legitimate complex constellation points associated with the specific modulation scheme employed. Based on Equation (7), the solution vector found by the GA is given by:

$$
\hat{\mathbf{x}}_{\mathrm{GA}}=\arg \left\{\min _{\tilde{\mathbf{x}} \in \mathcal{M}^{m_{t}}}[\Omega(\check{\mathbf{x}})]\right\},
$$

which is the individual having the lowest objective score (i.e. the OF's output) in terms of GA terminology. With the aid of the GA's natural evolution-like search mechanism, the GA-based detection technique is capable of achieving nearoptimum performance at a significantly lower complexity than that imposed by the full-search based ML detection. For more details on GA-based detection, such as the population initialization, fitness evaluation, individual selection, crossover, mutation, and elitism processes, the interested readers are referred to references [2], [3], [6]. Here we point out that the GA-aided detection schemes found in the open literature [2][6] can only provide a GA-individual based hard-decoded solution, which inevitably limits the GA-aided system's attainable performance. Furthermore, none of these previous schemes considered the so-called overloaded scenarios, where the number of transmit antennas is higher than the number of receiver antennas. In Section IV, the proposed GA with the ability to provide soft outputs will be introduced, which is capable of performing well even in overloaded systems.

\section{GAs Using Population-Based Soft Output}

In this section we derive an algorithm that enables the GA to output soft information. The soft-bit value or Log-Likelihood Ratio (LLR) associated with the $\left(m_{B}\right)^{\text {th }}$ bit position at the $j^{\text {th }}\left(j=1, \ldots, m_{t}\right)$ transmit antenna can be formulated as [10]:

$$
\mathcal{L}_{j, m_{B}}=\ln \frac{P\left(b_{j, m_{B}}=1 \mid \mathbf{y}, \mathbf{H}\right)}{P\left(b_{j, m_{B}}=0 \mid \mathbf{y}, \mathbf{H}\right)},
$$

which is the natural logarithm of the quotient of probabilities that the bit considered has a value of $b_{j, m_{B}}=1$ or $b_{j, m_{B}}=0$. Here we have omitted again the indices of $[n, k]$. Note that the probability $P\left(b_{j, m_{B}}=b \mid \mathbf{y}, \mathbf{H}\right)$ that the symbol transmitted by the $j^{\text {th }}$ transmit antenna has the $m_{B}^{\text {th }}$ bit value of $b_{j, m_{B}}=$ $b \in\{0,1\}$, is given by the sum of all the probabilities of the symbol combinations which assume that $b_{j, m_{B}}=b$. Hence, Equation (8) can be equivalently rewritten as:

$$
\mathcal{L}_{j, m_{B}}=\ln \frac{\sum_{\check{\mathbf{x}} \in \mathcal{M}_{j, m_{B}, 1}^{m_{t}}} P(\check{\mathbf{x}} \mid \mathbf{y}, \mathbf{H})}{\sum_{\check{\mathbf{x}} \in \mathcal{M}_{j, m_{B}, 0}^{m_{t}}} P(\check{\mathbf{x}} \mid \mathbf{y}, \mathbf{H})},
$$

where $\mathcal{M}_{j, m_{B}, b}^{m_{t}}$ denotes the specific subset associated with the $j^{\text {th }}$ transmit antenna, which is constituted by those specific trial vectors, whose $j^{\text {th }}$ element's

$$
\begin{aligned}
& \mathcal{M}_{j, m_{B}, b}^{m_{t}}= \\
& \left\{\check{\mathbf{x}}=\left(\check{x}_{1}, \ldots, \check{x}_{m_{t}}\right)^{T} \mid\left\{\check{x}_{1}, \ldots, \check{x}_{m_{t}} \in \mathcal{M}_{c}\right\} \wedge\left\{b_{j, m_{B}}=b\right\}\right\} .
\end{aligned}
$$

With the aid of Bayes' theorem [10], we have:

$$
P(\check{\mathbf{x}} \mid \mathbf{y}, \mathbf{H})=P(\mathbf{y} \mid \check{\mathbf{x}}, \mathbf{H}) \frac{P(\check{\mathbf{x}})}{P(\mathbf{y})} .
$$

Upon substituting Equation (11) into Equation (9), we arrive at:

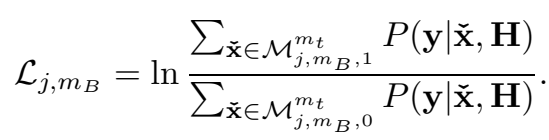


Note that here we have assumed that the different $\left(2^{m}\right)$ ary symbol combination vectors $\check{\mathrm{x}}$ have the same probability, namely that $P(\check{\mathbf{x}}), \check{\mathbf{x}} \in \mathcal{M}_{c}$ is a constant. On the other hand, it can be observed from Equation (2) that $\mathbf{y}$ is a random sample of the $m_{t}$-dimensional multi-variate complex Gaussian distribution, where the mean vector is $\mathbf{H x}$, while the $\left(n_{r} \times n_{r}\right)$ dimensional covariance matrix $\mathbf{R}_{\mathbf{w}}$ is given by [9]:

$$
\mathbf{R}_{\mathbf{w}}=E\left\{\mathbf{w w}^{H}\right\}=\sigma_{w}^{2} \mathbf{I}
$$

where $\sigma_{w}^{2}$ is the noise variance and $\mathbf{I}$ is the identity matrix, while the noise encountered at the $n_{r}$ number of receiver antennas is assumed to be uncorrelated. Hence, the abovementioned multi-variate complex Gaussian distribution can be described by [11]:

$f(\mathbf{y} \mid \mathbf{x}, \mathbf{H})=\frac{1}{\pi^{n_{r}}\left|\mathbf{R}_{\mathbf{w}}\right|} \exp \left\{-(\mathbf{y}-\mathbf{H} \mathbf{x})^{H} \mathbf{R}_{\mathbf{w}}^{-\mathbf{1}}(\mathbf{y}-\mathbf{H x})\right\}$.

When substituting Equation (13) into Equation (14), we have:

$$
f(\mathbf{y} \mid \mathbf{x}, \mathbf{H})=\frac{1}{\pi^{n_{r}} \sigma_{w}^{2}} \exp \left\{-\frac{1}{\sigma_{w}^{2}}\|\mathbf{y}-\mathbf{H x}\|^{2}\right\} .
$$

Note that $f(\mathbf{y} \mid \mathbf{x}, \mathbf{H})=P(\mathbf{y} \mid \mathbf{x}, \mathbf{H})$ is the a priori probability that the vector $\mathbf{y}$ has been received under the condition that the vector $\mathbf{x}$ was transmitted over the MIMO channel characterized by the CHTF matrix $\mathbf{H}$. Thus, Equation (12) can be further developed with the aid of Equation (15), yielding:

$$
\mathcal{L}_{j, m_{B}}=\ln \frac{\sum_{\check{\mathbf{x}} \in \mathcal{M}_{j, m_{B}, 1}^{m_{t}}} \frac{1}{\pi^{n_{r} \sigma_{w}^{2}}} \exp \left\{-\frac{1}{\sigma_{w}^{2}}\|\mathbf{y}-\mathbf{H} \check{\mathbf{x}}\|^{2}\right\}}{\sum_{\check{\mathbf{x}} \in \mathcal{M}_{j, m_{B}, 0}^{m_{t}}} \frac{1}{\pi^{n_{r} \sigma_{w}^{2}}} \exp \left\{-\frac{1}{\sigma_{w}^{2}}\|\mathbf{y}-\mathbf{H} \check{\mathbf{x}}\|^{2}\right\}} .
$$

In order to avoid the exponential computation imposed by Equation (16), the maximum-approximation [9] can be applied, yielding:

$$
\mathcal{L}_{j, m_{B}} \approx-\frac{1}{\sigma_{w}^{2}}\left[\left\|\mathbf{y}-\mathbf{H} \check{\mathbf{x}}_{j, m_{B}, 1}\right\|^{2}-\left\|\mathbf{y}-\mathbf{H} \check{\mathbf{x}}_{j, m_{B}, 0}\right\|^{2}\right]
$$

where

$$
\check{\mathbf{x}}_{j, m_{B}, b}=\arg \left\{\min _{\check{\mathbf{x}} \in \mathcal{M}_{j, m_{B}, b}^{m_{t}}}\left[\|\mathbf{y}-\mathbf{H} \check{\mathbf{x}}\|^{2}\right]\right\}, \quad b=\{0,1\} .
$$

Furthermore, with the aid of Equation (7), Equation (17) can be represented as:

$$
\mathcal{L}_{j, m_{B}} \approx-\frac{1}{\sigma_{w}^{2}}\left[\bar{\Omega}_{j, m_{B}, 1}-\bar{\Omega}_{j, m_{B}, 0}\right]
$$

where

$$
\bar{\Omega}_{j, m_{B}, b}=\min \left[\Omega\left(\check{\mathbf{x}}_{j, m_{B}, b}\right), \omega\right], \quad b=\{0,1\},
$$

and $\omega=m_{t} n_{r}$ is a normalization factor. Equation (18) suggests that the LLRs can be obtained by evaluating the GA's OF. More explicitly, in order to calculate the LLR of the $\left(m_{B}\right)^{t h}$ bit of the $j^{t h}\left(j=1, \ldots, m_{t}\right)$ transmit antenna at the specific subcarrier considered, the $X$ number of individuals in the GA's final generation are divided into two groups, where

\begin{tabular}{|c|c|c|}
\hline \multirow{6}{*}{$\begin{array}{l}\text { TC code } \\
\text { parameters }\end{array}$} & Modem & QPSK \\
\hline & Code rate & $1 / 2$ \\
\hline & Compnent codes & $\overline{\mathrm{RSC}}$ \\
\hline & Constraint length & 3 \\
\hline & $\begin{array}{l}\text { Octal generator poly- } \\
\text { nomial }\end{array}$ & $\left(\begin{array}{ll}7 & 5\end{array}\right)$ \\
\hline & $\begin{array}{ll}\text { Turbo } & \text { interleaver } \\
\text { length } & \\
\end{array}$ & 124 bits \\
\hline \multirow{10}{*}{$\begin{array}{c}\text { GA } \\
\text { parameters }\end{array}$} & $\begin{array}{l}\text { Population } \\
\text { initialization method }\end{array}$ & MMSE \\
\hline & $\begin{array}{l}\text { Mating pool creation } \\
\text { strategy }\end{array}$ & $\begin{array}{l}\text { Pareto- } \\
\text { Optimality [15] }\end{array}$ \\
\hline & Selection method & $\begin{array}{l}\text { Fitness- } \\
\text { Proportionate }\end{array}$ \\
\hline & Cross-over & $\begin{array}{l}\text { Uniform cross- } \\
\text { over }\end{array}$ \\
\hline & Mutation & BQM [7] \\
\hline & Elitism & Enabled \\
\hline & Incest prevention & Enabled \\
\hline & Population size & 500 \\
\hline & Generations & 5 \\
\hline & Mutation probability & 0.1 \\
\hline \multirow{6}{*}{$\begin{array}{l}\text { Channel } \\
\text { parameters }\end{array}$} & CIRs & BUG [14] \\
\hline & Paths & 8 \\
\hline & Maximum path delay & $40 \mu \mathrm{s}$ \\
\hline & Symbol duration & $160 \mu \mathrm{s}$ \\
\hline & Subcarriers & 128 \\
\hline & $\begin{array}{l}\text { Channel interleaver } \\
\text { length }\end{array}$ & 248 bits \\
\hline
\end{tabular}
the first (or second) group is constituted by those individuals that have a value of one (or zero) at the $\left(m_{B}\right)^{t h}$ bit of the $j^{t h}$ transmit antenna. The resultant lowest $\mathrm{OF}$ score calculated in each of the two groups is then compared to $\omega$, and the smaller
TABLE I

Basic Simulation Parameters Used in Section V

of the two will be used in Equation (18) for calculating the corresponding LLR, which can therefore assist the channel decoder in improving the SDM-OFDM system's performance.

It is worth pointing out that the proposed population-based GA only imposes a modest complexity increase in comparison to the conventional hard-decision aided individual-based GAs [2]-[6]. This is because the only additional operation required by the proposed scheme is to compare $\omega$ to the objective scores, which are already available, since the results of the OF evaluation carried out by the conventional GAs can be readily used.

\section{Simulation Results}

The OFDM modem used in our simulations employed 128 subcarriers. The half-rate TC [12] code employed two Recursive Systematic Convolutional (RSC) component codes having a constraint-length of $K=3$ and the standard 124bit Wideband CDMA (WDMA) Universal Mobile Telecommunications System's (UMTS) turbo code interleaver of [13]. The octally represented RSC generator polynomial of $\left(\begin{array}{ll}7 & 5\end{array}\right)$ was used. The 8-path dispersive fading channel model of [14] was employed and the channel estimation was assumed to be perfect. The Minimum Mean Square Error (MMSE) algorithm was used for creating the GA's initial population and the Biased $Q$-function based Mutation (BQM) scheme of [7] was employed. QPSK was used for all simulations. For the reader's convenience, the simulation parameters are summarized in Table I. For more details on the GA's configuration, the interested reader is referred to [2], [6], [7]. 


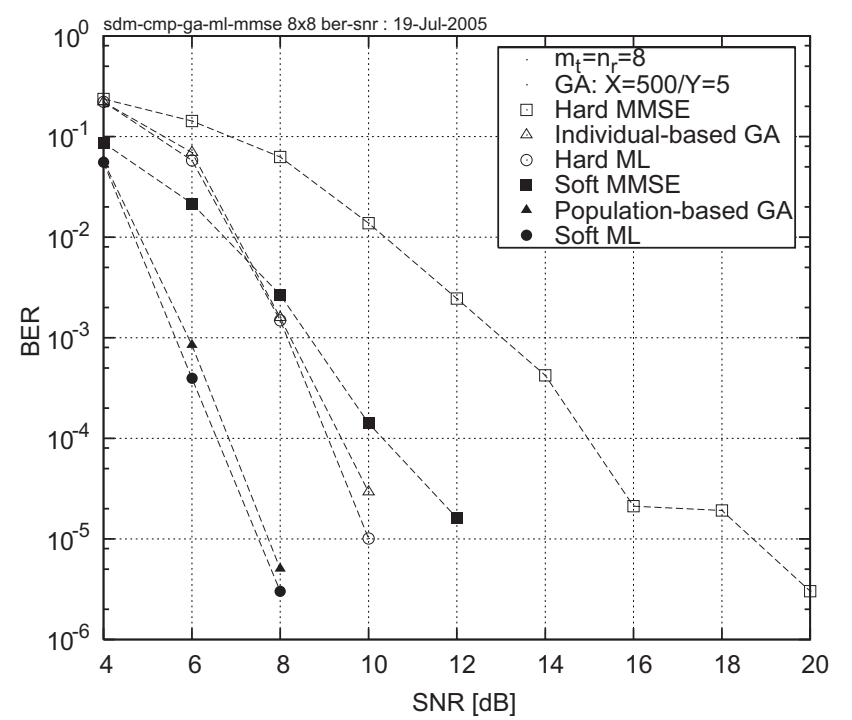

Fig. 2. BER performance comparison of the 8-receiver TCSDM-OFDM system using the individual-based hard-decoded and population-based soft-output GA detection, while employing a QPSK scheme having an effective throughput of 8 BPS.

Fig. 2 provides the Bit Error Rate (BER) performance of the TC-aided GA-SDM-OFDM system employing both the conventional individual-based hard-decoded GA solution [2], [4]-[7] and the proposed GA using population-based soft information, respectively. For the sake of benchmarking, the BER performances of the systems using hard-decoded and/or soft-information aided MMSE and/or ML detection are also shown in Fig. 2, where we had $m_{t}=n_{r}=8$ for all schemes, implying an effective throughput of $\left(2 \cdot 8 \cdot \frac{1}{2}\right)=8$ BPS. It can be seen in Fig. 2 that the GA-aided system was capable of achieving a similar BER performance to that attained by the optimum ML-aided arrangement, while outperforming the linear MMSE detected system. Furthermore, an $E_{b} / N_{0}$ gain of about $2 \mathrm{~dB}$ was achieved by the GA using population-based soft outputs over its counterpart using the individual-based hard-decoded outputs. In Fig. 3 we provide the BER performance recorded in the overloaded scenario, where $m_{t}=8$ transmit antennas and $n_{r}=6$ receiver antennas were employed. In overloaded scenarios, the weight matrix calculated by the MMSE algorithm becomes a singular matrix, which will lead to a theoretically unresolvable detection problem and thus resulting in a dramatic performance degradation, as shown in Fig. 3. By contrast, the system aided by the GA providing soft-outputs was capable of attaining an undistinguishable performance from that of the optimum soft-ML detected arrangement in the overloaded scenario of Fig. 3. Again, a $2 \mathrm{~dB} E_{b} / N_{0}$ gain was observed, when comparing the proposed GA to the GAs found in the literature [2], [4]-[6].

Furthermore, the associated complexity of the proposed GA-aided detection is significantly lower than that of the ML detection, as observed in Fig. 4, where we had $m_{t}=6$ or $m_{t}=8$ transmit antennas and $n_{r}=6$ receiver antennas. The associated complexity was quantified in terms of the number of complex additions and multiplications imposed by the different arrangements on a per user basis. As seen

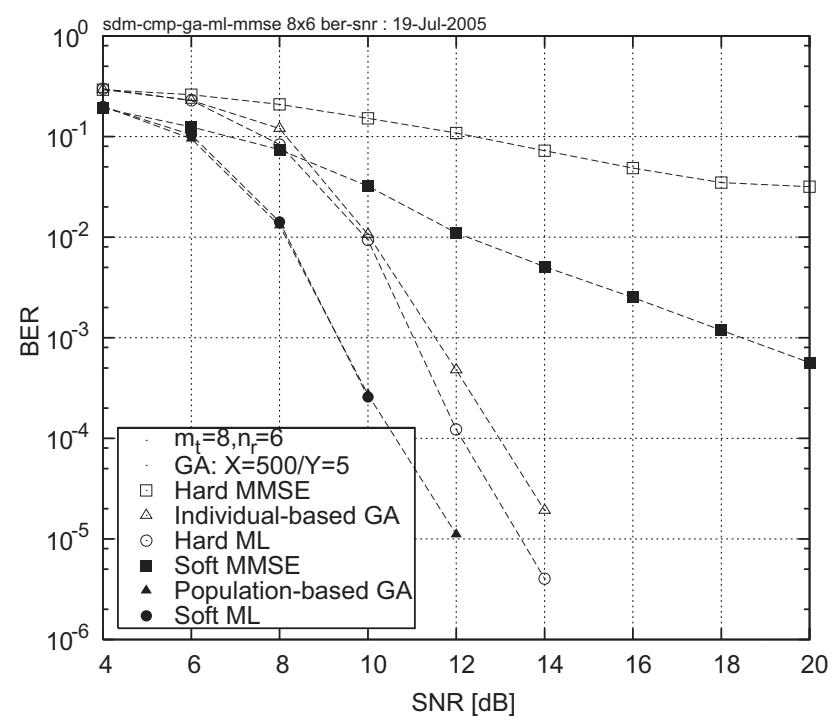

Fig. 3. BER performance comparison of the 6-receiver TCSDM-OFDM system using the individual-based hard-decoded and population-based soft-output GA detection, while employing a QPSK scheme having an effective throughput of 8 BPS.

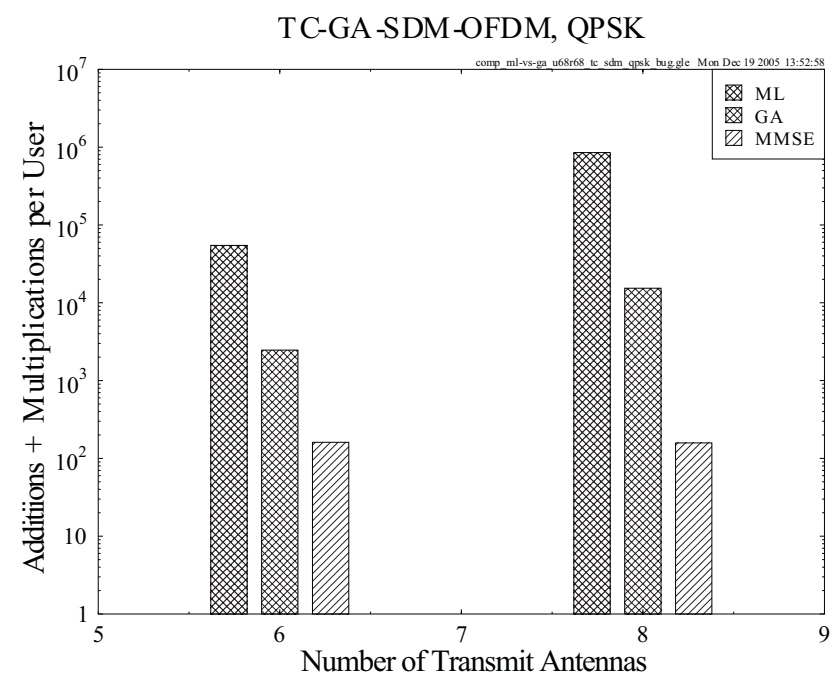

Fig. 4. Complexity per user versus number of transmitter antennas performance comparison of the ML, GA and MMSE assisted detection schemes. The number of receiver antennas used was $n_{r}=6$.

in Fig. 4, on one hand, the GA-aided scheme imposed a significantly lower complexity than that of the ML-aided arrangement. More precisely, the complexity reduction factors achieved by the GA-aided detection in comparison to the ML detection were in excess of 22 and 55, respectively, when we had $m_{t}=6$ and $m_{t}=8$ transmit antennas. On the other hand, although the complexity of MMSE detection is lower than that of the GA-aided detection, the performance of the former is substantially worse than that of the latter, especially in the overloaded scenario associated with Fig. 3. Explicitly, the GA is capable of achieving a near-optimum performance at the cost of a modest complexity, striking an attractive performance-versus-complexity design trade-off. 


\section{Conclusions}

In conclusion, the performance of the TC-SDM-OFDM system using the individual-based hard-decoded GA's output can be improved by about $2 \mathrm{~dB}$, when the proposed populationbased soft-output GA is employed. Furthermore, the GAdetected TC-SDM-OFDM system is capable of achieving an undistinguishable performance to that of the optimum soft-ML detected arrangement, while reducing the ML complexity by a factor of 55 for $m_{t}=8$. When a higher number of transmit antennas is used, the achievable complexity reduction becomes even more significant.

\section{REFERENCES}

[1] J. Holland, Adaptation in Natural and Artificial Systems. Ann Arbor, Michigan: University of Michigan Press, 1975.

[2] L. Hanzo, L.-L. Yang, E.-L. Kuan, and K. Yen, Single- and MultiCarrier DS-CDMA: Multi-User Detection, Space-Time Spreading, Synchronisation and Standards. New York: IEEE Press - John Wiley \& Sons Ltd., 2003.

[3] C. Ergün and K. Hacioglu, "Multiuser detection using a genetic algorithm in CDMA communications systems," IEEE Trans. Commun., vol. 48, no. 8, pp. 1374-1383, Aug. 2000.

[4] K. Yen and L. Hanzo, "Antenna-diversity-assisted genetic-algorithmbased multiuser detection schemes for synchronous CDMA systems," IEEE Trans. Commun., vol. 51, no. 3, pp. 366-370, Mar. 2003.

[5] - "Genetic-algorithm-assisted multiuser detection in asynchronous CDMA communications," IEEE Trans. Veh. Technol., vol. 53, no. 5, pp. 1413-1422, Sep. 2004.
[6] M. Jiang and L. Hanzo, "Genetically enhanced TTCM assisted MMSE multi-user detection for SDMA-OFDM," in Proc. IEEE 60th Veh. Technol. Conf., Fall, Sep. 2004, vol. 3, pp. 1954-1958.

[7] M. Jiang, S. X. Ng, and L. Hanzo, "Hybrid iterative multi-user detection for channel coded space division multiple access OFDM systems," IEEE Trans. Veh. Technol., vol. 55, no. 1, pp. 115-127, Jan. 2006.

[8] G. J. Foschini, "Layered space-time architecture for wireless communication in a fading environment when using multi-element antennas," Bell Labs Technical J., pp. 41-59, Autumn 1996.

[9] L. Hanzo, M. Münster, B. J. Choi, and T. Keller, OFDM and MC-CDMA for Broadband Multi-User Communications, WLANs and Broadcasting. New York: IEEE Press - John Wiley \& Sons Ltd., 2003.

[10] T. K. Moon and W. C. Stirling, Mathematical Methods and Algorithms for Signal Processing. Upper Saddle River, NJ: Prentice Hall, 2002.

[11] S. Kay, Fundamentals of Statistical Signal Processing, Estimation Theory. Upper Saddle River, NJ: Prentice Hall, 1993.

[12] L. Hanzo, T. H. Liew, and B. L. Yeap, Turbo Coding, Turbo Equalisation and Space-Time Coding for Transmission Over Fading Channels. New York: IEEE Press - John Wiley \& Sons Ltd., 2002.

[13] H. Holma and A. Toskala, WCDMA for UMTS: Radio Access for Third Generation Mobile Communications, 3rd ed. New York: John Wiley \& Sons, Ltd, 2004.

[14] S. Bug, C. Wengerter, I. Gaspard, and R. Jakoby, "Channel modeling based on comprehensive measurements for DVB-T mobile applications," in Proc. 18th IEEE Instrumentation Measurement Technol. Conf., May 2001, vol. 1, pp. 544-548.

[15] D. E. Goldberg, Genetic Algorithms in Search, Optimization, and Machine Learning. Reading, MA: Addison-Wesley, 1989. 